\title{
HEAD EXTENSION IS ESSENTIAL FOR ADEQUATE VENTILATION THROUGH LARYNGEAL TUBE
}

\author{
Manpreet Singh ${ }^{1}$, Rajesh Singh Rautela ${ }^{2}$, Surendra Kumar ${ }^{3}$ \\ Assistant Professor, Government Medical College Hospital, Sec 32, \\ Chandigarh, India ${ }^{1}$, Professor/ MD/ UCMS and GTB Hospital, Dilshad Garden, Delhi, India ${ }^{2}$, Specialist/ \\ MD/ UCMS and GTB Hospital, Dilshad Garden, Delhi, India ${ }^{3}$ \\ *Corresponing Email- manpreetdawar@hotmail.com
}

Key words: Laryngeal tube; Head Extension

Experience of 3 years of repeated use of LT in more than 270 cases conducted under general anaesthesia in all age groups,it was keenly observed that extension of head maneuver is usually required for adequate ventilation through laryngeal tube in more than $95 \%$ of cases.

The laryngeal tube (VBM Medizintechnik, Sulz, Germany) is a relatively new supraglottic device, designed to secure a patent airway during either spontaneous breathing or controlled ventilation ${ }^{(1)}$.The manufacturer's standard LT was designed and later on modified with incorporation of suction port. This standard LT consists of an airway tube with a small cuff attached at the tip (distal cuff) and a larger balloon cuff at the middle part of the tube (Proximal cuff). The cuffs are inflated through a single pilot tube and balloon, through which pressure can be monitored. There are 3 black lines on tube near a standard $15 \mathrm{~mm}$ connector, which indicate adequate depth of insertion when aligned with teeth. The device is inserted while patient's head and neck are placed either in the sniffing position or in neutral position. The tip of a well lubricated LT is placed against the hard palate behind the upper incisors. The device is then slid down the centre of the mouth until resistance is felt or the device is almost fully inserted. When the laryngeal tube is inserted properly, the second bold black line on the tube should have just passed between upper and lower incisor teeth. When ventilation is adequate, a bite block is inserted; the LT is fixed in place with an elastic strap. A correctly placed LT will maintain its intended position after insertion and it is fixed with bite block and elastic strap provided with LT. The correct placement is confirmed by effective mechanical ventilation $^{(2)}$, capnography and auscultation over areas of chest bilaterally.

We have experience of 3 years of extensive use of LT for at least 270 cases conducted under general anaesthesia in all age groups. The device is used routinely to maintain airway either during spontaneous breathing or controlled ventilation. Most often (>95\% cases) it is noted that extension of head maneuver is usually required for adequate ventilation (Fig 1A).

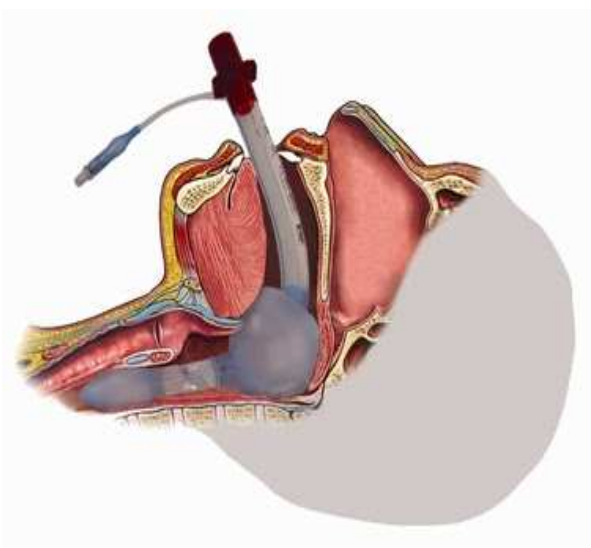


If this maneuver is not performed, there is loss of canographic waveform, chest expansion decreases and patients become hypoxic. A detailed study of equipment was done and it was noted that this can be due to improper sealing of LT cuff with pharyngeal mucosa. This seal is adequately formed when the head of patient remains extended throughout intraoperative period and there is perfect alignment of ventilation port and larynx. This axis and alignment is absolutely necessary for ventilation.

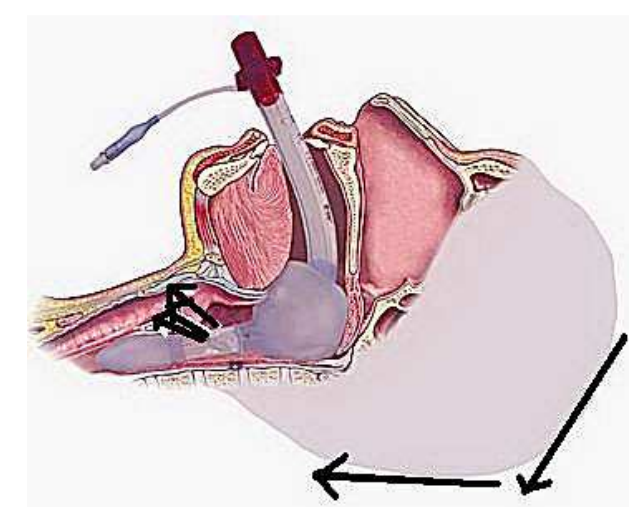

The head can be fixed in extended position by use of a thinner donate or a circular sandbag with thickness that fits in the neck region.It can also be fixed with microtape such that the head remains immobile once the position is made. Inadequate positioning of head during intraoperative period leads to improper ventilation and transient hypoxia. This can be rectified by head extension maneuver and this helps in motivating the anaesthesiologist for using this supraglottic device fearlessly.

\section{References}

1. Asai T, Shingu K.The laryngeal tube. Br J Anaesth 2005 Dec; 95 (6):729-36.

2. Gaitine LA, Vaida SJ, Somri M, etal. An evaluation of the laryngeal tube during general anesthesia using mechanical ventilation. Anesth Analg 2003; 96:1750-5.

\section{Legends}

Take in Figure 1- shows laryngeal tube insitu

Take in Figure 2 shows laryngeal tube in extension of head with alignment of ventilation port with laryngeal opening 